\title{
DYNAMIC INSTABILITY OF A HOMOGENOUS DEFORMATION OF A THIN ELASTIC BAR*
}

\author{
BY TIMOTHY J. BURNS (Sandia National Laboratories†, Albuquerque)
}

\begin{abstract}
A linear stability analysis of a homogeneous deformation at constant strainrate of a thin elastic bar is used to show that the deformation is unstable with respect to small perturbations in the case when the stress-strain relation is concave with a single maximum.
\end{abstract}

1. Introduction. The one-dimentional thin elastic bar is a convenient model for the study of the instability of a deformation in a material with a concave constitutive relation $\sigma(\varepsilon)$ with a single maximum at $\varepsilon=\varepsilon_{c}$, where $\sigma$ is the stress and $\varepsilon$ is the strain $[2,7]$. Such instabilities have been observed in experiments at constant strain-rate in which thin-walled metal tubes loaded in torsion begin to form one or more shear bands at strains near or exceeding $\varepsilon_{c}$, the critical strain at which the maximum of $\sigma(\varepsilon)$ occurs $[4,5]$. In this study, a linear stability analysis is used to show that small perturbations on a homogeneous longitudinal extension, at constrant strain-rate $\dot{\varepsilon}_{0}$, of a thin elastic bar with a concave constitutive relation, do not grow catastrophically in amplitude until the bar has been strained to near or beyond the critical strain $\varepsilon_{c}$. Further, if a perturbation is separated into normal modes, then the modes corresponding to the longest wavelengths can grow the most. Once the critical strain has been exceeded, however, small perturbations can grow exponentially in amplitude.

2. Dynamic elastic bar deformation. The motion of a one-dimensional thin elastic bar is governed by the partial differential equation (see, e.g., [6, Ch. II, §1])

$$
\rho_{\mathrm{o}} \frac{\partial^{2} u}{\partial t^{2}}=\frac{\partial \sigma}{\partial X}
$$

where $X \in[0, L], L>0$, is the Lagrangian material coordinate; $t \in[0, T], T>0$, is the time; $\rho_{0}>0$ is the constant initial density at each point $X$ in the bar; and $u(X, t)$ is the displacement at time $t$ of the material particle $X$. The stress $\sigma$ and the strain $\varepsilon=\partial u / \partial X$ are assumed to satisfy a constitutive relation $\sigma=\sigma(\varepsilon)$, with $\sigma \in C^{2}\left[0, \varepsilon_{1}\right]$, for some $\varepsilon_{1}>0$, such that $\sigma(\varepsilon) \geq 0$ and $\sigma(0)=0 ; d \sigma / d \varepsilon>0$ for $\varepsilon \in\left[0, \varepsilon_{c}\right), d \sigma / d \varepsilon=0$ for $\varepsilon=\varepsilon_{c}$, and $d \sigma / d \varepsilon<0$ for $\varepsilon \in\left(\varepsilon_{c}, \varepsilon_{1}\right]$, for some $0<\varepsilon_{c}<\varepsilon_{1}$. It is also assumed that there exist positive constants $a<b$ and $c<d$ such that

$$
-b \leq d^{2} \sigma / d \varepsilon^{2} \leq-a \text { for } \varepsilon \in\left[0, \varepsilon_{c}\right], \quad-d \leq d^{2} \sigma / d \varepsilon^{2} \leq-c \text { for } \varepsilon \in\left[\varepsilon_{c}, \varepsilon_{1}\right] .
$$

* Received October 26, 1981. This work was supported by the U.S. Department of Energy under Contract DE-AC04-76-DP00789. The author would like to thank D. E. Grady for suggesting this problem and for several discussions pertaining to this work. The author would also like to thank J. A. Ellison for a discussion of some of the ideas in Sec. 3 and T. G. Trucano and S. T. Montgomery for reviewing the manuscript.

$\dagger$ A U.S. Department of Energy facility. 
A simple but interesting non-steady solution of (1) is given by the homogeneous deformation of the bar at constant strain-rate with the point at $X=0$ held fixed,

$$
u_{h}(X, t)=\dot{\varepsilon}_{0} X t,
$$

where $\dot{\varepsilon}_{0}=\partial^{2} u_{h} / \partial X \partial t$ is the constant strain-rate, on the time interval $[0, T]$, with $T=$ $\varepsilon_{1} \dot{\varepsilon}_{0}^{-1}$.

3. Linear stability analysis. The first variation of the bar equation (1) with respect to the homogeneous solution (3), obtained by linearizing Eq. (1) about (3) with $u=u_{h}+v$ for small $v$, is given by

$$
\partial^{2} v / \partial t^{2}=f(t) \partial^{2} v / \partial X^{2}
$$

where $f(t)=\left.\left(1 / \rho_{0}\right)(d \sigma / d \varepsilon)\right|_{\varepsilon=\varepsilon_{0} t}$, so that $f(t)>0$ for $t \in\left[0, t_{c}\right), f\left(t_{c}\right)=0$, and $f(t)<0$ for $t \in\left(t_{c}, T\right]$, where $t_{c}=\varepsilon_{c} \varepsilon_{0}^{-1}$. Note that Eq. (4) changes type from hyperbolic to elliptic at $t=t_{c}$. A small perturbation of the homogeneous solution satisfies (4) to first order, with boundary conditions

$$
v\left(0, t_{0}\right)=v\left(L, t_{0}\right)=0, \text { for some } 0<t_{0}<T,
$$

small initial amplitude

$$
v\left(X, t_{0}\right)=H(X), \text { for some } H(X) \in C^{2}[0, L], \quad \text { with } H(0)=H(L)=0,
$$

and small initial velocity

$$
\frac{\partial v}{\partial t}\left(X, t_{0}\right)=K(X) \text {, for some } K(X) \in C^{2}[0, L], \text { with } K(0)=K(L)=0 .
$$

The method of separation of variables leads to a representation of the solution of the initial-boundary value problem in terms of a Fourier sine series on $\left[t_{0}, T\right]$,

$$
v(X, t)=\sum_{k=1}^{\infty} V_{\beta}(t) \sin \beta X, \quad \beta=\pi k L^{-1},
$$

which is in $\left.C^{2}(0, L] \times\left[t_{0}, T\right]\right)$. Each term in (5) is a solution of the variational equation (4) which satisfies the periodic boundary conditions. Substitution of such a normal-mode solution into (4) leads to an ordinary differential equation for the growth of the amplitude of each term in the sine series,

$$
\ddot{V}+\beta^{2} f(t) V=0,
$$

where the subscript $\beta$ has been dropped and a dot denotes $d / d t$, with initial values $V\left(t_{0}\right)=$ $V_{0}, \dot{V}\left(t_{0}\right)=\dot{V}_{0}$ determined by the coefficients in the sine series expansions of $H(X)$ and $K(X)$ on $[0, L]$. As is common in fluid dynamics stability theory (see, e.g., [8, §27]), the homogeneous solution will be called stable if the amplitude functions $V_{\beta}(t)$ do not grow in absolute value on the finite time interval $\left[t_{0}, T\right]$, and unstable otherwise.

The behavior of solutions of the amplitude equation (6) will be studied by comparison with solutions of the well-known Airy equation,

$$
d^{2} W / d \tau^{2}-\tau W=0 .
$$

It will be shown that every solution of $(6)$ on the interval $[0, T]$ must oscillate a finite number of times on the subinterval $\left[0, t_{c}\right]$ and remain bounded in terms of its initial values, 
while for $t \in\left[t_{c}, T\right]$, solutions of (6) can grow exponentially in absolute value. From the convexity condition (2), it follows that there exists a positive constant $A$, which depends on the constant $a$ in (2) and on the constant strain-rate $\dot{\varepsilon}_{0}$, such that, for $t \in\left[0, t_{c}\right], f(t) \geq$ $A\left(t_{c}-t\right)$. Let $g(t)=A\left(t_{c}-t\right)$, and consider the equation

$$
\ddot{Z}+\beta^{2} g(t) Z=0 .
$$

The change of independent variable $\tau=\beta^{2 / 3} A^{1 / 3}\left(t-t_{c}\right)=-(\beta / A)^{2 / 3} g(t)$ on $\left[0, t_{c}\right)$ transforms Eq. (8) into the Airy equation (7), with $\tau \in\left[-\tau_{1}, 0\right]$, where $\tau_{1}=\beta^{2 / 3} A^{1 / 3} t_{c}$. Hence, $Z(t)=W(\tau)$ for $t \in\left[0, t_{c}\right]$ whenever the two solutions and their derivatives have the same initial values at some point in the interval. Since $f(t) \geq g(t)$ on $\left[0, t_{c}\right]$, it follows from the Sturm comparison theorem [3, Ch. 2, Th. 6] that a nontrivial solution of (6) must vanish at least once between any two zeros of a nontrivial solution of (8). It is well known (see, e.g., [1, \$37]) that all solutions of (7) oscillate for $\tau<0$, so that any solution of (6) must oscillate at least a finite number of times (zero times is allowed) on $\left[0, t_{c}\right]$. A similar argument using (2) to bound $f(t)$ from above by a linear function on $\left[0, t_{c}\right]$ shows that no nontrivial solution of (6) can oscillate more than a finite number of times on $\left[0, t_{c}\right]$, for otherwise this would also be true for a nontrivial solution of (7).

Next, let $V(t)$ denote a nontrivial solution of (6) on $\left[t_{0}, T\right]$ for some $0<t_{0}<t_{c}$. Then substituting $V(t)$ into $(6)$, multiplying through by $\dot{V}(t)$, and integrating by parts yields

$$
\frac{1}{2}[\dot{V}(t)]^{2}+\frac{\beta^{2}}{2} f(t)[V(t)]^{2}=\frac{1}{2} \dot{V}_{0}^{2}+\frac{1}{2} \beta^{2} f\left(t_{0}\right) V_{0}^{2}+\frac{\beta^{2}}{2} \int_{t_{0}}^{t} f^{\prime}(s)[V(s)]^{2} d s .
$$

Hence, it follows that, since $f^{\prime}(t)$ is negative on $\left[t_{0}, t_{c}\right)$,

$$
0 \leq \frac{\beta^{2}}{2} f(t)[V(t)]^{2}-\frac{\beta^{2}}{2} \int_{t_{0}}^{t} f^{\prime}(s)[V(s)]^{2} d s \leq \frac{1}{2} \dot{V}_{0}^{2}+\frac{\beta^{2}}{2} f\left(t_{0}\right) V_{0}^{2} .
$$

Let $V_{m}=\max \left\{|V(t)|: t \in\left[t_{0}, t_{c}\right]\right\}$. Then

$$
\frac{\beta^{2}}{2} f(t) V_{m}^{2}-\frac{\beta^{2}}{2}\left[f(t)-f\left(t_{0}\right)\right] V_{m}^{2}=\frac{\beta^{2}}{2} f\left(t_{0}\right) V_{m}^{2} \leq \frac{1}{2} \dot{V}_{0}^{2}+\frac{\beta^{2}}{2} f\left(t_{0}\right) V_{0}^{2} .
$$

By Bessel's inequality, for all $\beta$,

$$
\dot{v}_{0}^{2} \leq \frac{2}{\pi} \int_{0}^{\pi}[K(X)]^{2} d X \equiv J,
$$

so it follows that, for all $\beta$,

$$
0 \leq \frac{1}{2} \beta^{2} f\left(t_{0}\right)\left[V_{m}^{2}-V_{0}^{2}\right] \leq \frac{1}{2} J .
$$

The inequality (10) implies that $V_{m}^{2}-V_{0}^{2}$ decreases to zero as $\beta$ increases to infinity, so that on the subinterval $\left[t_{0}, t_{c}\right]$, perturbations corresponding to the longest wavelengths can grow the most, with the maximum growth bounded by $J\left[\beta^{2} f\left(t_{0}\right)\right]^{-1}$. Since $f(t)$ decreases to zero as $t$ increases to $t_{c}$, it follows that $V_{m}^{2}$ can be larger the closer $t_{0}$ is to $t_{c}$.

For $t \in\left[t_{c}, T\right], f(t)$ is less than or equal to zero, so a corollary to the Sturm comparison theorem [3, Ch. 2, $\$ 4$, Cor. 1] implies that the nontrivial solutions of (6) can have at most one zero on $\left[t_{c}, T\right]$. For the above solution $V(t)$, let this zero, if it exists, be denoted by $t_{1}$, 
and assume that $t_{1}>t_{c}$ and that $V(t)$ is negative on $\left[t_{c}, t_{1}\right)$ and positive on $\left(t_{1}, T\right]$. Then the substitution $W=\dot{V} V^{-1}$ transforms (6) into the following Riccati equation:

$$
\dot{W}+W^{2}+\beta^{2} f(t)=0,
$$

on the two subintervals $\left[t_{c}, t_{1}-\delta\right]$ and $\left[t_{1}+\delta, T\right]$, for any sufficiently small $\delta>0$. Using the convexity relations (2) again, bounds will next be obtained on the growth of $W(t)$, the logarithmic derivative of $V(t)$, which will then lead to bounds on the growth of $V(t)$ on $\left[t_{c}\right.$, $T]$ in terms of the well-known Airy functions $\mathrm{Ai}(t)$ and $\mathrm{Bi}(t)$, which are two linearly independent solutions of the Airy equation (7).

Condition (2) implies that, for $t \in\left[t_{c}, T\right]$, there exist positive constants $C$ and $D$, with $C<D$, which depend on the constants $c$ and $d$ in (2) and on the constant strain-rate $\dot{\varepsilon}_{0}$, such that

$$
-D\left(t-t_{c}\right) \leq f(t) \leq-C\left(t-t_{c}\right) .
$$

Let $h(t)=C\left(t-t_{c}\right)$ and $k(t)=D\left(t-t_{c}\right)$. It follows that, on the two subintervals $\left[t_{c}, t_{1}-\delta\right]$ and $\left[t_{1}+\delta, T\right]$,

$$
-W^{2}+\beta^{2} h(t) \leq \dot{W} \leq-W^{2}+\beta^{2} k(t) .
$$

Let $\left.W_{1}=\dot{V} t_{c}\right)\left[V\left(t_{c}\right)\right]^{-1}$ and $W_{2}=\dot{V}\left(t_{1}+\delta\right)\left[V\left(t_{1}+\delta\right)\right]^{-1}$, and consider the two Riccati equations

$$
\dot{R}+R^{2}-\beta^{2} h(t)=0, \quad \dot{S}+S^{2}-\beta^{2} k(t)=0 .
$$

Because of the bounds (12) on $W(t)$, it follows from a standard comparison theorem [3, Ch. $1, \S 12$, Th. 8] that

$$
R(t) \leq W(t) \leq S(t)
$$

on $\left[t_{c}, t_{1}-\delta\right]$ and $\left[t_{1}+\delta, T\right]$, with $R\left(t_{c}\right)=S\left(t_{c}\right)=W_{1}$ on the first subinterval and $R\left(t_{1}+\delta\right)=S\left(t_{1}+\delta\right)=W_{2}$ on the second subinterval. Now, $W(t)$ is the logarithmic derivative of $V(t)$, so that, on $\left[t_{c}, t_{1}-\delta\right]$,

$$
\exp \left[\int_{t_{c}}^{t} R(\tau) d \tau\right]-W_{1} \leq V(t)\left[V\left(t_{c}\right)\right]^{-1} \leq \exp \left[\int_{t_{c}}^{t} S(\tau) d \tau\right]-W_{1}
$$

and on $\left[t_{1}+\delta, T\right]$,

$$
\exp \left[\int_{t_{c}}^{t} R(\tau) d \tau\right]-W_{2} \leq V(t)\left[V\left(t_{1}+\delta\right)\right]^{-1} \leq \exp \left[\int_{t_{c}}^{t} S(\tau) d \tau\right]-W_{2} .
$$

But $\exp \left[\int_{t_{c}}^{t} R(\tau) d \tau\right]$ and $\exp \left[\int_{t_{c}}^{t} S(\tau) d \tau\right]$ are solutions of the second-order equations

$$
\ddot{P}-\beta^{2} h(t) P=0, \quad \text { and } \quad \ddot{Q}-\beta^{2} k(t) Q=0,
$$

which can be transformed into the Airy equation (7) by means of a linear change of independent variable, as was done above with Eq. (8). Since $|V(t)|$ can be made arbitrarily small on $[-\delta, \delta]$ for sufficiently small $\delta>0$ by continuity, it follows that the solution $V(t)$ of (6) is bounded above and below by linear combinations of the Airy functions $\mathrm{Ai}(\tau)$ and $\operatorname{Bi}(\tau)$, where $\tau$ is an increasing linear function of $t$, and the two constants in the linear combination are determined by $V\left(t_{c}\right)$ and $\dot{V}\left(t_{c}\right)$ on $\left[t_{c}, t_{1}-\delta\right]$ and by $V\left(t_{1}+\delta\right)$ and 
$\dot{V}\left(t_{1}+\delta\right)$ on $\left[t_{1}+\delta, T\right]$. It is well-known that $\mathrm{Ai}(\tau)$ has the leading behavior $\operatorname{Ai}(\tau) \sim$ $\pi^{-1 / 2} \tau^{-1 / 4} \exp \left[-2 \tau^{3 / 2} / 3\right]$ as $\tau \rightarrow \infty$ and $\mathrm{Bi}(\tau)$ has the leading behavior $\mathrm{Bi}(\tau) \sim \pi^{-1 / 2} \tau^{-1 / 4}$ $\exp \left[2 \tau^{3 / 2} / 3\right]$ as $\tau \rightarrow \infty$ (see, e.g., $[1, \S 3.5$, Ex. 5]). Since, for fixed $t, \tau \rightarrow \infty$ as $\beta \rightarrow \infty$ (see the discussion following Eq. (8)), it follows that most perturbations of the homogeneous solution (3) of (1), i.e. all except those whose amplitudes depend only on $\mathrm{Ai}(\tau)$ or only on small values of $\beta$, grow exponentially in amplitude on $\left[t_{c}, T\right]$. The cases where a solution of (6) has no zero on $\left[t_{c}, T\right]$ or is positive on $\left[t_{c}, t_{1}\right)$ and negative on $\left(t_{1}, T\right]$, forsome $t_{1}$ satisfying $t_{c}<t_{1}<T$, lead to essentially the same conclusion. Hence, the homogeneous solution (3) is an unstable solution of the bar equation (1).

\section{REFERENCES}

[1] C. M. Bender and S. A. Orszag, Advanced mathematical methods for scientists and engineers, McGraw-Hill, New York, 1978

[2] B. Bernstein and L. J. Zapas, Stability and cold drawing of viscoelastic bars, J. Rheology 25 (1), 83-94 (1981)

[3] G. Birkhoff and G. C. Rota, Ordinary differential equations, 2nd ed., Blaisdell, Waltham, 1969

[4] T. J. Burns, D. E. Grady, and L. S. Costin, On a criterion for thermoplastic shear instability, Amer. Inst. Phys. Conf. Ser. No. 78, Chapt. 7, 372-375 (1981)

[5] L. S. Costin et al., On the localization of plastic flow in mild steel tubes under dynamic torsional loading, Amer. Inst. Phys. Conf. Ser. No. 47, Chapt. 1, 90-100 (1979)

[6] N. Cristescu, Dynamic plasticity, John Wiley \& Sons, New York, 1967

[7] J. L. Ericksen, Equilibrium of bars, J. Elasticity 5, 191-201 (1975)

[8] L. D. Landau and E. M. Lifschitz, Fluid mechanics, Pergamon, Oxford, 1959 